Cahiers Société

\title{
Note de recherche. Dialectique de l'aliénation, I. La critique du sujet
}

\section{François L'Italien}

Numéro 1, mai 2019

URI : https://id.erudit.org/iderudit/1068427ar

DOI : https://doi.org/10.7202/1068427ar

Aller au sommaire du numéro

Éditeur(s)

Collectif Société

ISSN

2562-5373 (imprimé)

2562-5381 (numérique)

Découvrir la revue

Citer cette note

L'Italien, F. (2019). Note de recherche. Dialectique de l'aliénation, I. La critique du sujet. Cahiers Société, (1), 207-218. https://doi.org/10.7202/1068427ar d'utilisation que vous pouvez consulter en ligne. 


\section{| Recensions et notes critiques}

\section{Dialectique de l'aliénation I. La critique du sujet}

François L'ITALIEN Institut de recherche en économie contemporaine (IREC)

Depuis la fin du siècle dernier, la tradition phénoménologique a inspiré parmi les plus puissantes relectures philosophiques de l'œuvre de Marx. Ces relectures ont profondément renouvelé le regard porté sur cette pensée ainsi que sur ses objets de préoccupation, et ce, au moment où s'épuisaient les variantes " orthodoxes » du marxisme. Du Marx de Michel Henry publié en 1976 jusqu'à aujourd'hui, les hypothèses fondamentales de la phénoménologie ont été mises de l'avant pour réfléchir et approfondir l'œuvre marxienne en un sens inédit.

Quel est ce sens? Ces relectures ont vu dans cette œuvre une rupture radicale avec l'hégélianisme et ses présupposés, au nom du fait que cette philosophie s'inscrirait pleinement dans le mouvement ayant mené au déploiement planétaire $d u$ capitalisme, à l'émancipation de la technique et à la domination des machines. Figure achevée de la métaphysique occidentale, l'hégélianisme constituerait en réalité le versant onto-philosophique d'un puissant vecteur de rationalisation totalisante du réel ayant pris naissance en Grèce antique. Marx aurait compris qu'une rupture s'imposait aussi bien avec la philosophie hégélienne qu'avec le capitalisme et la forme-valeur, pour autant où ils représenteraient des manifestations d'un seul et même processus d'auto-objectivation du rationnel, processus s'identifiant au réel lui-même. Ainsi, là où les marxismes critiques entendaient dans les Thèses sur Feuerbach un appel au renouvellement de la dialectique, ces réinterprétations soutiennent au contraire que Marx y énoncerait les grandes lignes d'une conception distincte de la pratique. En fait, Marx aurait cherché à penser la pratique et le monde autrement que sur le mode hégélien d'une logique d'objectivation de soi du sujet dans l'histoire, que ce dernier soit compris avec ou sans majuscule.

Ces réinterprétations de l'œuvre de Marx sont à méditer aussi bien pour la justesse de certaines thèses qu'elles avancent sur cette œuvre, que pour ce qu'elles donnent à penser sur notre condition actuelle. Si cette identification du sujet et, plus largement, 
de la dialectique de la raison, au déploiement historique du techno-capitalisme est problématique à plusieurs égards, elle soulève cependant des questions d'ordre sociologique et philosophique qui méritent d'être abordées de front. Les développements qui suivent sont animés de la conviction que la pensée dialectique a avantage à prendre au sérieux ces relectures phénoménologiques de Marx, dans la mesure où il y a là une occasion de penser avec elles les apories du système social contemporain mais aussi, et plus largement, l'« énigme » de l'Occident ${ }^{1}$.

Prolongeant en l'infléchissant une réflexion initiée il y a quelques années autour de l'interprétation henryenne de $\mathrm{Marx}^{2}$, le présent texte expose et questionne une de ces relectures significatives, soit celle proposée par Franck Fischbach. Sans être très récente, cette réinterprétation est encore proche de nous, puisqu'elle soulève des problèmes philosophiques, sociologiques et épistémologiques fondamentaux qui traversent les traditions de pensée. S'appuyant plus spécifiquement sur Heidegger pour relire Marx, des excursions dans l'œuvre du premier sont menées par Fischbach pour mieux comprendre et en quelque sorte prolonger la pensée du second. Comme nous le verrons, ces rapprochements sont justifiés pour plusieurs raisons ; à commencer par celle où ces deux penseurs, faisant l'expérience de la crise de la modernité à des moments distincts, ont vu dans la radicalité des transformations opérées par le techno-capitalisme les effets d'une aliénation fondamentale, celle d'une perte ou d'une privation de monde, située au cœur de la civilisation occidentale. À tous deux s'est présentée l'urgente nécessité de penser l'agir sur la base de ses modalités premières d'appartenance et de participation au monde, de le soustraire au règne du capital et du devenir-machine de l'humanité afin de maintenir ouverte la possibilité d'un « autre commencement ». L'objectif ici sera d'examiner de manière critique les propositions avancées par Marx et Heidegger, telles qu'elles ont été récapitulées et synthétisées par Fischbach, dans l'optique de contribuer à une théorisation dialectique de l'aliénation contemporaine.

\section{Sujet et aliénation}

Dans deux ouvrages complétant un triptyque portant sur la philosophie marxienne, soit Sans objet3 et La privation de monde4, Fischbach établit un dialogue entre Heidegger et Marx sur la base de leur commune détermination à rompre avec les philosophies modernes du sujet. Pensée à travers plusieurs écrits sans jamais avoir

\footnotetext{
${ }^{1}$ Jacques-Alexandre Mascotto, « Michel Freitag : principe civilisationnel contre civilizational fracking du capital et du système américain », dans La liberté à l'épreuve de l'histoire. La critique du libéralisme chez Michel Freitag, dir. Daniel Dagenais, Montréal, Liber, 2017, p. 361-402.

${ }^{2}$ François L'Italien, "La praxis comme production de la vie», dans Marx philosophe, dir. Olivier Clain, Montréal, Nota Bene, 2009 ; Praxis et subjectivité. Contribution à la critique de la philosophie de la pratique de Marx, Mémoire de Maîtrise, Université Laval, 2004.

${ }^{3}$ Franck Fischbach, Sans objet. Capitalisme, subjectivité, aliénation, Paris, Vrin, 2009.

${ }^{4}$ Franck Fischbach, La privation de monde. Temps, espace et capital, Paris, Vrin, 2011.
} 
été exposée systématiquement chez Marx, cette rupture est mise en lumière en recourant à Heidegger, qui explicite les linéaments d'une pensée "alternative » du soi et du monde. Pour Fischbach, nulle autre catégorie que celle de l'aliénation ne pouvait mieux révéler la bifurcation qu'a prise la pensée de Marx vis-à-vis de ces philosophies du sujet, et c'est sur la base d'un examen de la signification et de la portée de cette catégorie qu'il entend montrer la nature de la critique adressée par Marx à la plus redoutable d'entre elles, celle de Hegel.

Rappelons d'abord des choses connues. Classiquement, l'aliénation renvoie à une problématique typiquement moderne, reposant sur le principe d'une opposition du sujet avec le monde. Depuis Descartes, en effet, le sujet est la catégorie désignant une substance réfléchissante faisant face à une altérité, le monde, s'opposant à elle comme une substance étendue, prenant la forme de l'objet. Les manières de penser cette opposition entre le sujet et l'objet, mais aussi et surtout le dépassement de cette opposition, ont été nombreuses, singulièrement dans l'idéalisme allemand. Le fait est que cette opposition laissait ouverte la possibilité que le sujet se perde dans l'objet, y soit absorbé et s'oublie en lui, à défaut de le faire sien et de s'y reconnaître. Hegel est certainement celui qui a proposé la manière la plus appropriée de résoudre cette opposition: dépassant les limites de l'entendement d'un sujet fini, Hegel a fait de l'opposition du sujet et de l'objet, ainsi que de la sortie de soi du premier vers le second, la structure même de la processualité-dialectique immanente au réel. Dans cette processualité, qui est le véritable Sujet, « il faut entendre que l'autre apparemment objectif du sujet est en fait toujours le résultat d'une objectivation de soi du sujet, d'un devenir-autre à soi tel que l'autre du sujet est en fait toujours en même temps son autre, à partir duquel il lui est possible de faire retour à soi5. » Hegel appelle aliénation ce processus d'extériorisation ou de sortie de soi (Entfremdung) du sujet, extériorisation par laquelle l'objet advient finalement comme une détermination du sujet qui, au départ, lui échappait comme telle. Ainsi comprise, l'aliénation est un processus propre à l'essence dialectique du réel, par lequel l'étrangeté du monde est progressivement abolie par son identification à la trajectoire du processus substantiel qui est Sujet.

Or, on le sait, c'est ce mouvement dialectique de sortie et de réintégration de soi qui a posé problème aux successeurs et héritiers «critiques » de Hegel : le dépassement dynamique de l'opposition sujet/objet au sein de la processualité-dialectique du Sujet a été dénoncé sous le chef qu'il s'agissait là d'un dépassement strictement théorique, laissant le réel aux prises avec sa scission interne. Non seulement ce processus d'objectivation inconsciente de soi du sujet devait être rapporté à l'activité réelle de sujets sociaux plutôt qu'à l'autodéveloppement d'un Sujet hypostasié, mais il pouvait, dans la pratique, être bloqué ; les mouvements d'extériorisation de soi des sujets sociaux pouvaient ne pas être suivis d'une réintériorisation des déterminations de ce

\footnotetext{
${ }^{5}$ Franck Fischbach, Sans objet, op. cit., p. 39.
} 
monde, et ainsi mener à un étrangement progressif vis-à-vis d'eux-mêmes et du monde. Le thème de la réification chez Lukács, où le sujet se voit imposer le mode d'être des objets qu'il a pourtant lui-même produit, est l'expression achevée de cette redéfinition de l'aliénation, comprise désormais comme la condition d'un sujet social non seulement privé de sa capacité à réintégrer ses objectivations, mais éventuellement dominé par elles.

Si Marx partagera au départ cette critique jeune-hégélienne de l'aliénation et du problème de l'opposition du sujet à l'objet, il réalisera rapidement que cette critique partage avec Hegel des présupposés communs, qui sont ceux d'une philosophie où la pratique, la vie en commun et le monde sont encore compris et unifiés par le biais des catégories de sujet et d'objet, de conscience de soi et de rapport d'objectivation. Or, Fischbach montre que, dès les Manuscrits de 18446, Marx engage une rupture avec ces présupposés et développe une position philosophique distincte du « soi », du monde, ainsi que de l'aliénation. Dans ces manuscrits, Marx avance que l'aliénation, loin d'être la perte ou la capture du sujet dans l'objet, loin d'être l'imposition du mode d'être de l'objectivité de l'objet à la subjectivité du sujet, consiste d'abord et avant tout à définir l'être humain comme un sujet. Se concevoir a priori comme sujet, c'est-à-dire en relation avec un monde d'objets extérieurs qui existent en face et séparément de ce sujet, se concevoir comme une substance qui est a priori étrangère à l'objectivité du monde, c'est là en réalité le résultat même de l'aliénation. Pour Marx, considérer l'être humain comme un sujet suppose qu'une dimension constitutive de son être lui ait été arrachée et retranchée, de telle sorte qu'il en soit réduit à être une substance qui soit a priori opposée à l'objectivité du monde. Dans les $\mathrm{Ma}$ nuscrits de 1844, Marx appelle «objectivité » cette dimension constitutive de l'être de l'être humain, dimension dont l'expropriation ou la perte définit l'essence même de l'aliénation. L'aliénation consiste dans le fait d'être "sans objet» (gegenstandlos), de perdre ses objets propres et, conséquemment, d'être réduit à la condition privative d'un sujet.

Ainsi, contrairement aux présupposés des philosophies modernes, le sujet n'est pas l'alpha et l'oméga de la réalité, mais bien l'expression d'une condition sociohistorique donnée, qui a été produite par une dépossession essentielle : l'être humain est d'abord et avant tout un être objectif, à qui l'on a précisément retiré son objectivité. Cette objectivité se décline de plusieurs manières, qui peuvent se résumer en deux principales. D'abord, l'être humain est un être objectif puisqu'il est doté de déterminations sensibles qui le rattachent au reste de la nature. L'homme, dit Marx, est une « part de la nature », en ce qu'il participe d'un monde lui ayant dispensé une sensibilité et des pouvoirs, mais aussi des affects et des besoins qu'il doit combler. Être objectif, c'est faire partie de cette trame naturelle à travers des formes, qui spécifient

\footnotetext{
6 Karl Marx, Les manuscrits économico-philosophiques de 1844, Paris, Vrin, 2007. Cette nouvelle traduction réalisée et présentée par Franck Fischbach s'ouvre sur un exposé synthétique de la théorie de l'aliénation chez Marx.
} 
cette appartenance à la vie sensible et au monde. Ensuite, l'être humain est un être objectif en ce qu'il tend naturellement à objectiver son propre être, au sens aussi bien utilitaire qu'expressif du terme. La production (producere) d'un monde est une disposition propre à l'être humain, qui doit, pour réaliser son être, s'accomplir dans des œuvres et des outils. L'homme, ne cesse de répéter Marx, est un «tool-making animal », un être qui, pour accomplir son essence, doit s'objectiver à travers des techniques et des produits par lesquels il participe à ce monde. Il s'agit, là aussi, d'une dimension essentielle ou « objective » du mode d'être de l'humanité.

Ce n'est que lorsque ces dimensions objectives de l'être humain lui sont retirées que ce dernier s'appauvrit et déchoit au rang de sujet, c'est-à-dire au rang d'être privé d'objectivité. Aux yeux de Marx, cette condition de sujet est celle de l'aliénation, qui est survenue de manière massive avec l'irruption du capitalisme dans l'histoire. Basé sur l'expropriation de l'objectivité matérielle propre à l'être humain, le capitalisme est un régime ayant dépossédé les individus des moyens ainsi que des fruits de leur activité pratique :

dépourvu d'accès direct à la matière de son travail, dépossédé des moyens objectifs de son travail comme des outils de sa production, exclu de la jouissance du produit de son propre travail, l'homme est réduit à la figure aliénée de lui-même en tant que pur sujet, simple possesseur d'une force purement subjective de travail à laquelle ont été soustraites toutes les conditions objectives de sa propre actualisation?.

Cela permet de comprendre la nature de la critique qu'adresse Marx, dès 1844-1845, aux philosophies du sujet: plutôt que de partir de l'opposition entre le sujet et le monde des objets, et de chercher à identifier les voies d'un raccordement entre eux comme le fait Hegel, il convient plutôt de rendre compte des conditions dans lesquelles l'être humain a été privé de ses objets essentiels pour devenir un sujet défini par une intériorité séparée du monde. En transcendantalisant la catégorie de sujet, les philosophies modernes ont, à l'instar de l'économie politique classique face au travail, escamoté la genèse historique de cette condition. En en faisant le principe actif derrière l'activité du connaître et de l'agir, ces philosophies ont dissimulé cette aliénation en dés-historicisant la catégorie de sujet, et en élevant la séparation dont il est l'effet au rang d'ontologie. Aucun autre philosophe n'a plus contribué à cela que Hegel, qui a fait du Sujet la figure «motrice» du devenir rationnel du cosmos, de l'être jusqu'à l'État constitutionnel moderne. Or, cette séparation, dit Marx, n'est pas le point de départ : c'est le point d'arrivée d'un processus de dépossession qu'il s'agit d'expliquer plutôt que de recouvrir.

\footnotetext{
${ }^{7}$ Ibid., p. 50.
} 
Nous devons à Franck Fischbach d'avoir recomposé la critique marxienne de l'aliénation, identifiant les philosophies du sujet au développement du capitalisme. Cette relecture a le mérite d'exhiber un ressort essentiel de la philosophie de Marx que la tradition critico-dialectique avait mis de côté. Car il est vrai qu'à partir de 1844, et ce jusqu'aux écrits sur le capital, Marx a délaissé les présupposés des philosophies du sujet pour développer une autre voie ; pour parler de la pratique et de la vie en commun, Marx va en effet recourir aux catégories de force de travail vivante, de travailleur subjectif ou de subjectivité vivante pour traiter de la pratique, et réserver celles de Sujet, d'universel et d'objectivité pour aborder les processus de renforcement de l'expropriation de l'être objectif dans le capitalisme. Si Marx reconnaîtra toute sa vie sa dette à l'égard de la dialectique hégélienne, tout semble indiquer que cette dette ne renvoyait pas tant à une fidélité à l'égard de la primauté du sujet et rapport d'objet, qu'à celle où le réel est d'abord et avant tout compris comme contradiction et conflictualité. La dialectique marxienne, tout comme le socialisme, constituerait la voie d'un autre commencement, en dehors du cosmos de la rationalité bourgeoise s'exprimant aussi bien dans la philosophie du sujet que dans le capital.

Cette critique marxienne de l'aliénation a thématisé des ruptures qui ont été reprises, de différentes manières, par les courants postmodernes au $\mathrm{XX}^{\mathrm{e}}$ siècle qui ont cherché à liquider le sujet. Peut-on dire, pour cela, que Marx a été l'un des premiers penseurs postmodernes ? Serait-il plutôt le premier penseur moderne à théoriser la postmodernité ? La question est ouverte. Plutôt que de trancher cela par un jugement de Salomon, il importe plutôt de relever ici brièvement des angles morts de la critique marxienne du sujet afin d'en déterminer les limites. Cela permettra ensuite d'approfondir la réalité de l'être objectif identifiée par Marx, réalité qu'aurait mieux décrite phénoménologiquement Heidegger.

La première limite a trait à la caractérisation de l'objectivité de l'être humain par Marx. S'il est vrai que les philosophies modernes du sujet ont transcendantalisé cette catégorie, c'est d'abord parce qu'elles ont énoncé le principe cardinal de la lutte menée par la société bourgeoise contre l'Ancien régime, principe qui était celui de la liberté et de la réflexivité politique. Contre un ordre traditionnel «arbitrairement» justifié sur la base du droit divin et du lignage, la bourgeoisie a fait valoir la nécessité d'asseoir les normes sur la possibilité d'en démontrer et d'en débattre la rationalité. Pour le dire simplement, il ne devait plus y avoir de « sujets » du roi, dont la pratique était politiquement déterminée sans pouvoir être déterminante, mais bien plutôt des sujets égaux devant la loi, lesquels accepteraient d'être contraints par des normes pour autant où ils pouvaient agir sur les règles présidant à leur élaboration.

La mise en place d'un système d'institutions légitimées sur la base de la liberté politique a complété le renversement de l'antique signification du sujet, pour établir l'appartenance au monde et à la société à un niveau supérieur. Être sujet de la loi, c'était avant tout appartenir aux défenseurs de la liberté politique, qui formaient ensemble, autour de cette valeur supérieure dotée d'un haut degré d'abstraction subs- 
tantielle, une communauté de destin. En cela, et contrairement à ce qu'affirment Marx et Fischbach, il faut dire que la condition de sujet politique a maintenu et enrichi l'objectivité de l'être humain, dans la mesure où la loi et les institutions politiques constituaient des objectivations substantielles, ayant pour finalité explicite d'exprimer l'humanité de l'être humain. Dans son principe, le sujet de la loi a constitué, dans la société moderne, l'étalon de mesure pour évaluer l'aliénation des sujets de la domination religieuse ou traditionnelle. Or, cet aspect de l'objectivité de l'être humain est évacué par Marx, qui est ambigu sur cette question. Dans le meilleur des cas, la pratique politique constitue pour lui une objectivation détenant un coefficient de réalité inférieur à celle des outils, des produits ou des œuvres; dans le pire des cas, la vie politique moderne participe de la désappropriation de l'être objectif en faisant de ce dernier un sujet de droits, formellement libre mais matériellement assujetti.

Corollairement à cela, si le capitalisme est né dans les pores de la société bourgeoise, comme le dit Marx, il est problématique d'inférer qu'il serait de la même nature qu'elle. Aussi, Marx et Fischbach laissent entendre, sur ce point qui est d'une importance épistémologique et politique décisive, que le capitalisme serait coextensif des institutions politiques modernes et radicaliserait en quelque sorte la rationalité de ces institutions en l'appliquant sans fard au domaine privé de l'organisation capitaliste. Cela permet ainsi d'identifier le processus de mise à nu des êtres sociaux enclenché par le capital, aux présupposés des philosophies du sujet, qui légitimeraient et renforceraient en quelque sorte cette privation de l'objectivité de l'être humain. En ce sens, la "modernité capitaliste » dont font état Marx et la relecture de Fischbach s'accorde parfaitement avec le libéralisme anglo-saxon, pour lequel les institutions politiques sont des émanations instrumentales des puissances de la société civile. Or, s'il est vrai que le capitalisme est né dans les pores de la société bourgeoise, il est davantage conforme à la dialectique historique réelle d'affirmer qu'il s'agissait là plutôt d'un corps étranger porteur d'une autre rationalité que celle qui a caractérisé les institutions politiques de la société moderne. La mise à nu subjective des salariés par l'expropriation des conditions de leurs activités et de leur savoir-faire dans la manufacture est d'une nature distincte du sujet de la loi, dont l'objectivité se manifeste et se réalise dans la vie publique. Pour le dire autrement, le Sujet hégélien, d'inspiration républicaine, dispose d'une objectivité sociale se situant aux antipodes du sujet libéral nu, dépossédé et délié du monde ${ }^{8}$. Théorisant quelques décennies à peine après que la chouette de Hegel eut récapitulé, à son crépuscule, le devenir du monde moderne, Marx a plutôt fait l'expérience de l'aube d'un autre monde, où le capital jouera une fonction déterminante dans l'aliénation de l'objectivité politique de l'être humain. Cette aliénation, qui grève aujourd'hui

\footnotetext{
${ }^{8}$ Pour approfondir cette distinction décisive, l'ouvrage de Michel Freitag, L'abîme de la liberté : critique du libéralisme, Montréal, Liber, 2011, portant sur la dialectique de la liberté en Occident, demeure à ce jour indépassé.
} 
l'humanité de l'homme, est bien celle-là dont il faut prioritairement faire la critique. Nous aurons l'occasion d'y revenir ultérieurement.

S'il est vrai que ces précisions n'invalident pas la thèse d'un étrangement progressif des individus à l'égard du monde en Occident, étrangement prenant racine dans l'ébranlement des certitudes premières face au cosmos vécu en Grèce ancienne et contre lequel Marx se dresse, elles viennent cependant établir le fait que la catégorie de sujet ne peut être définie comme une condition essentiellement aliénante. La prise en compte de la dialectique historique des derniers siècles permet d'identifier et d'approfondir des logiques de régulation distinctes entre les institutions de type moderne, reposant sur des sujets réputés être investis d'une raison substantielle par laquelle ils s'objectivent politiquement, et les organisations capitalistes postmodernes, produisant des subjectivités nues à travers un arraisonnement fonctionnel de leur pratique.

Ceci dit, la critique marxienne du sujet soulève un point qui nous semble essentiel à considérer sociologiquement et philosophiquement. Il s'agit des limites manifestes du monde moderne à pouvoir maintenir l'unité des dimensions subjectives et objectives de la pratique dans le contexte d'une institutionnalisation et d'une rationalisation poussées des domaines de la vie sociale. Si le sujet politique moderne appartenait désormais à "son » monde par le détour de médiations institutionnelles davantage que par la tradition ou la foi, il s'est avéré que cette modalité de l'être objectif présentait des limites anthropologiques importantes. Non seulement la société moderne devait-elle sans cesse susciter une "disposition subjective» (Hegel) nécessaire à l'adhésion des sujets aux institutions, mais l'institutionnalisation exubérante des domaines de la vie sociale a mis à l'épreuve, dans son mouvement historique, l'unité et le sens même du sujet politique. Ce devenir aporétique de la modernité politique a provoqué une fragmentation interne de son sens et la prolifération concomitante de subjectivités se rabattant sur les différentes facettes du «monde de la vie », lesquelles ont coïncidé avec l'expansion rapide et démesurée des organisations capitalistes en Occident, et bientôt sur la planète entière. Là, la critique du « subjectivisme » menée par Fischbach à travers Marx fait mouche, mais a le défaut d'identifier ce repli libéral sur soi à l'essence même du sujet moderne.

Contemporain des limites d'un projet dont l'unité reposait sur le sujet politique, et devant l'incapacité manifeste des sociétés à contenir l'expansion brutale de la logique d'expropriation de l'objectivité organisée par le capital, Marx a souhaité revenir en amont de cette objectivité et approfondir les ressorts premiers de l'appartenance, de l'agir et de la participation de l'être humain au monde. "Premiers », parce que ces ressorts seraient antérieurs à toute différenciation interne entre un sujet et un objet, et donc situés en amont de toute objectivation formelle, qu'elle soit de nature substantielle ou fonctionnelle. C'est ici que la mise en dialogue entre Marx et Heidegger administrée par Fischbach devient pertinente, puisqu'avec ce dernier il est possible de faire l'hypothèse que la pensée heideggérienne a prolongé et étoffé la catégorie 
d'être objectif proposée par Marx. Tout en refusant d'identifier le sujet et l'aliénation, et ce, en établissant comme nous l'avons fait des distinctions sociologiques essentielles, il semble nécessaire d'écouter la phénoménologie heideggérienne concernant cette modalité première de l'agir dans le monde, à laquelle la tradition dialectique a peu fait écho.

\section{Être-dans-le-monde et participation}

C'est dans Etre et temps de Heidegger que Fischbach trouve des matériaux susceptibles d'approfondir la compréhension d'une modalité première de participation, d'agir et d'accomplissement d'un « soi » dans le monde, un « soi » qui ne serait pas conçu comme une pure intériorité a priori séparée du monde. Cette modalité première est développée par Heidegger dans Être et temps à travers une phénoménologie de la quotidienneté de l'existant (Dasein), qui rompt explicitement avec les présupposés des philosophies du sujet. Pour Heidegger, en effet, il convient de rendre compte du fait que les choses dans la vie quotidienne ne se donnent jamais d'abord à l'existant de manière théorique, c'est-à-dire dans un "voir » impliquant une mise à distance avec elles. Ces dernières se donnent plutôt dans le commerce affairé que l'existant entretient avec le monde. Dans cet affairement, l'existant est en quelque sorte toujours déjà immergé et absorbé dans le monde, de telle manière qu'il ne se rapporte pas à lui comme à une instance séparée se déclinant en une série de phénomènes extérieurs : il est si imprégné du monde qu'il ne « voit » et n'objective pas la mondanéité du monde comme le ferait un sujet opposé à son objet.

Appelé à préciser cette modalité d'être de l'existant, Heidegger soutient que cet affairement dans le monde se réalise par l'usage pratique d'étants, qu'il nomme « outils». Les outils renvoient à tout étant susceptible d'être utilisé par l'existant pour faire monde ; les chaises, la table, la maison, mais aussi le soleil, l'air, la neige, sont des outils par lesquels l'existant se «mondanéise », pour ainsi dire. Dans l'usage qu'il fait de l'outil en vue de faire quelque chose, l'étant ne voit pas l'outil luimême ; l'outil se confond avec son usage et s'efface dans le «ce-en-vue-de-quoi » il doit être utilisé. Un pont est un chemin-pour-traverser la rivière, avant d'être un agencement de matériaux dotés de telle ou telle propriété physico-mécanique. Cet usage fait apparaître le monde comme une toile de renvois significatifs dans laquelle l'outil s'inscrit de manière immanente et dans laquelle l'existant est chez lui. Le monde est le nom donné à cette trame significative que fait apparaître l'usage pratique des outils par l'existant, et dans laquelle ce dernier est toujours déjà immergé.

Cette immersion quotidienne de l'existant dans l'usage est telle, soutient Heidegger, qu'il n'y a pas de "rapport» entre cet être et le monde, comme s'il s'agissait là de réunir deux réalités séparées. S'il y a bien des distinctions entre l'existant et le monde, il n'existe aucune séparation essentielle ou apriorique. Dans la vie quotidienne, l'existant est d'abord et avant tout un être-dans-le-monde, qui n'est 
pas confiné dans une intériorité séparée du reste : «L'être dans le monde est le mouvement d'une existence envoyée et expédiée dans le monde auprès des choses et des êtres dont elle s'occupe et qui la préoccupent ${ }^{9} »$. En fait, dit Fischbach, Heidegger va montrer que le sujet, loin d'être premier, est un mode dérivé de l'être de l'existant, un mode qui est concomitant du retrait de l'évidence du monde et de son apparition comme série d'objets. Ce n'est que lorsque le monde n'apparaît plus comme cette toile de renvois significatifs dans laquelle s'insère l'usage pratique des outils qu'il se retire comme monde et qu'apparaît concomitamment un sujet face à un objet.

Cette dissolution de la mondanéité du monde se traduit d'abord par une déchéance des étants, compris comme outils insérés dans la trame significative et matérielle du monde, au rang d'objets subsistants par eux-mêmes et en eux-mêmes, situés dans un espace et un temps formel et abstrait. Selon Heidegger, cette occultation de l' « êtrepour » des outils en entraîne une seconde : l'existant interprète son propre être à partir de sa relation aux étants subsistants, ce qui signifie qu'il s'interprète comme un sujet face à des étants subsistants devenus objets. Pour le dire autrement, ce n'est que lorsque l'évidence du monde se dissipe que, formellement, un rapport d'objectivation devient possible; ce n'est qu'à ce moment qu'une brèche décisive dans l'être de l'existant apparaît, brèche par laquelle ce dernier s'éloigne et devient étranger. Pour Fischbach, ce processus de mise à distance et de sortie hors du monde est précisément ce qui rapproche Heidegger du concept d'aliénation chez Marx : «L'aliénation est un éloignement à l'égard du monde ambiant conduisant à devenir étranger à la mondanéité même du monde quotidien de la préoccupation ${ }^{10}$. " En devenant étranger au monde, l'existant devient un sujet défini par son intériorité, et qui entretient désormais des rapports avec des objets étrangers.

Pour Heidegger, ce retrait de l'évidence du monde survient du moment où l'outil ne fonctionne plus, moment où il ne s'efface plus dans l'usage qu'il en est fait et par lequel l'existant se «mondanéise ». En dysfonctionnant, l'outil se détache en quelque sorte de la trame significative du monde pour être objectivé, et fait simultanément disparaître ce dernier. À cela il faudrait ajouter, avec Fischbach mais aussi Jean Vioulac ${ }^{11}$, que la perte du monde survient au moment où la pratique quotidienne décrite phénoménologiquement par Heidegger dans Être et temps devient elle-même problématique, comme dans le cas de la condition des salariés dans le capitalisme, qui sont dépossédés de leurs outils et des fruits de leur "mondanéisation ", qui ne cesse d'avorter. Avec le capitalisme vient le règne des machines et de la formevaleur, qui réduit l'existant au rang de subjectivité nue à laquelle fait face à un système d'objets lui étant de plus en plus étranger.

\footnotetext{
${ }^{9}$ Franck Fischbach, La privation de monde, op. cit., p. 53.

${ }^{10}$ Ibid., p. 57.

${ }^{11}$ Jean Vioulac, L'époque de la technique. Marx, Heidegger et la métaphysique, Paris, Presses Universitaires de France, 2014.
} 
Sans partager le diagnostic heideggérien associant l'apparition d'une fissure au sein de l'existant comme le motif initial d'un devenir historique culminant avec la planétarisation du capitalisme, l'émancipation de la technique et la philosophie hégélienne, il soulève cependant des questions importantes. D'une part, s'il est vrai que des différences existent entre la Raison substantielle de la tradition républicaine et la raison instrumentale de la tradition libérale, il est aussi juste de dire qu'elles ont des choses en commun. L'une d'entre elles est de reposer sur le principe de mise à distance et d'objectivation formalisée, principe dont le développement historique a en quelque sorte mis à l'épreuve la plasticité même du symbolique, dans sa capacité à maintenir les conditions d'un sens à travers les formes de plus en plus abstraites de son expression. L'Occident est la civilisation ayant expérimenté, avec les Grecs anciens, un ébranlement fondamental des évidences liées à la co-appartenance ontologique des sujets sociaux et du monde, un ébranlement ayant mené à une mise à distance des certitudes relatives au donné de la culture. La genèse de la critique comme discours et éventuellement comme pratique politique est ce qui a rendu possible la valorisation de l'objectivation formalisée dans le monde moderne, une valorisation qui a soutenu les deux grands courants de la rationalité moderne.

Il est ainsi pertinent de thématiser une modalité première d'être et d'appartenance au monde préalable à tout rapport d'objectivation proprement dit, une modalité fondamentale qui est devenue problématique. Si, contrairement à Marx et Heidegger, on ne peut affirmer que les philosophies du sujet, dont celle de Hegel, constituent l'expression idéologique de la perte du monde, il faut néanmoins reconnaître que ces philosophies ont supposé, à tort, que l'appartenance première au monde comportait une robustesse ontologique telle que son effectivité pouvait être resaisie et subsumée à travers des rapports d'objectivation de plus en plus abstraits. Ainsi, il ne fait pas de doute que la philosophie hégélienne ait pensé l'odyssée de la raison et du Sujet comme celle d'un approfondissement de l'appartenance de l'être social au monde, à son sens et à son « destin ». Mais il est cependant manifeste que cette compréhension de la raison et du sujet ait aussi reposé sur le présupposé selon lequel l'appartenance à ce monde pouvait se déployer dialectiquement et s'abstraire sans risque d'être diluée ou tout simplement laminée par des formes historiques censées l'enrichir. La crise du monde moderne a été l'expression historique d'un étrangement vis-à-vis du monde, un étrangement réfutant concrètement ce présupposé philosophique associant l'expérience du sujet à celle d'une refamiliarisation continue avec le monde. C'est, rappelons-le, l'un des constats sous-jacents à la conclusion de La condition de l'homme moderne de Hannah Arendt.

Ceci dit, il se trouve qu'une pensée des apories du monde moderne et des philosophies qui en ont récapitulé le principe de régulation essentiel a déjà été initiée, pensée qui a aussi thématisé dialectiquement le caractère ontologique et anthropologique de la modalité d'appartenance première au monde, aussi bien sur les plans épistémiques que socio-historique. C'est dans l'œuvre de Michel Freitag que l'on retrouve l'effort 
de penser les contours de l'expérience humaine du monde se rapprochant de ce que Marx et Heidegger ont cherché à mettre en lumière à travers les catégories d'être objectif, d'être-dans-le-monde et d'usage du monde. Pour Freitag, cette modalité première d'appartenance est celle de la culture, comprise au sens fort d'une manière d'habiter et de participer au monde, et qui présente un fort degré d'évidence phénoménologique. Cette modalité est précisément celle qui a été au cœur des sociétés de mythe et de parole, qui ont été aussi désignées comme des "sociétés de participation » par certains anthropologues, souhaitant par-là mettre en évidence qu'elles reposent sur « une structure d'appartenance ontologique réciproque de l'individu et de la société, de la société et de la nature, une appartenance qui est médiatisée par le langage et la culture et qui est alors indéfiniment diversifiée dans son unité12. » Afin de souligner toute la richesse de cette modalité de l'existence, Freitag a repris des Grecs anciens le concept d'ergazasthai, " qui exprimait encore cette condition synthétique de l'action comprise comme modalité humaine de participation avec la natu$\mathrm{re}^{13}$ ». Ce concept, rappelle Freitag, a longtemps renvoyé en Grèce à un état fondateur dans lequel l'ordre de la cité prenait la source de sa justification. C'est ainsi dans la resaisie de cette modalité originelle que les Grecs, et éventuellement certains courants modernes, ont pu chercher à légitimer la pratique et la connaissance, dans le souci de faire monde à travers ce détour. L'importance qu'a accordée Freitag à cette modalité de l'existence humaine dans son dernier ouvrage, L'abîme de la liberté, témoigne du fait qu'au-delà de son intérêt sociologique ou philosophique, elle comporte une dimension normative déterminante, c'est-à-dire susceptible d'éclairer le jugement que nous portons vis-à-vis de notre condition socio-historique actuelle.

Si nous faisons encore l'expérience quotidienne de cette modalité d'existence dans ce qu'il reste de culture au sens fort, elle se fraie un chemin jusque dans l'effectivité, lorsqu'apparaissent dans le débat public des questions touchant les fins de la vie collective. C'est dans ces débats, et surtout dans ce qu'il reste de capacités politiques à mettre en œuvre ces fins, que peut s'exprimer, malgré ses limites, cette appartenance au monde et où se joue notre condition humaine. En ce sens, le politique est, pour Freitag, le lieu où la civilisation de l'objectivation peut encore affirmer son « sens », c'est-à-dire l'histoire de cette co-appartenance des individus et du monde. Cela suppose que la critique de l'aliénation aujourd'hui doit s'accompagner d'une méditation sur les ressorts de notre participation et appartenance à cette Terre, sur les limites de l'objectivation formalisée quant à sa capacité à ressaisir l'essence de cet « engagement » au-delà d'un certain seuil d'abstraction, ainsi que sur les conditions à réunir pour défendre les capacités politiques des sociétés par lesquelles nous habitons ce monde.

\footnotetext{
12 Michel Freitag, «La nature de la technique », dans Michel Freitag (avec la collaboration d'Yves Bonny), L'oubli de la société. Pour une théorie critique de la postmodernité, Québec/Rennes, Presses de l'Université Laval/Presses Universitaires de Rennes, 2002, p. 340.

${ }^{13}$ Ibid., p. 342.
} 